\title{
Managing anemia in lymphoma and multiple myeloma
}

\author{
Gunnar Birgegård \\ Department of Haematology, \\ University Hospital, Uppsala, Sweden
}

\begin{abstract}
Anemia is common in cancer, and lymphoproliferative disease is no exception. Erythropoiesis-stimulating agents (ESA) have been used for renal anemia since 1986, and considerably later in cancer anemia. The first studies were published around 1993, but the use of ESA did not become common in cancer anemia until in the late 1990s. Cancer anemia is still under-treated. This review gives an overview of the use of ESA in hematologic malignancies. A background is given about this treatment in the cancer field generally. The pathophysiology of cancer anemia is described with special emphasis on the disturbances in iron metabolism. Functional iron deficiency has been shown to be both frequent and important as a hindrance for response to ESA treatment, and recent studies are reported in some detail, where the use of intravenous iron was shown to improve the response rate of ESA treatment.
\end{abstract}

Keywords: Epo, epoetin, ESA, cancer anemia, lymphoma, myeloma, lymphoproliferative disease

\section{Background}

Cancer patients may be anemic for a variety of reasons. It is good clinical practice always to bear in mind that other etiologies than the cancer itself may be at hand, even though the most common causes of anemia in general, like iron deficiency, are not very prevalent in hematological malignancies. However, exclusion of deficiency of iron, folate, and B12, as well as immunologically induced hemolytic anemia, should be part of the work-up of anemia even in this situation.

In general, anemia is common in lymphoproliferative disorders (lymphoma and multiple myeloma). Kyle (1975) reported $\mathrm{Hb}<12 \mathrm{~g} / \mathrm{dl}$ in $73 \%$ of newly diagnosed myeloma patients and Moullet and colleagues (1998) found $\mathrm{Hb}<11 \mathrm{~g} / \mathrm{dl}$ in 32\% of newly diagnosed non-Hodgkin's lymphoma patients. In a more recent study, the European Cancer Anaemia Survey (ECAS) (Ludwig et al 2004; Birgegård et al 2006) enrolled 2360 lymphoma and myeloma patients: $52.5 \%$ were anemic at enrolment and $73 \%$ showed a $\mathrm{Hb}<12 \mathrm{~g} / \mathrm{dl}$ during a 6-month follow-up period (Figure 1). As explained below, erythropoiesis is inhibited by complex mechanisms connected to the cancer itself. However, a close connection to cytostatic treatment has also been established. During CHOP (cyclophosphamide, doxorubicin, vincristine, prednisone) treatment $79 \%$ of the patients developed mild to moderate anemia and $49 \%$ World Health Organization (WHO) grade 3-4 anemia in a study by Groopman and Itri (1999). In the ECAS study, chemotherapy was the strongest factor contributing to anemia. In another multi-centre study it was found that the prevalence of anemia with $\mathrm{Hb}$ levels between 8 and $10.5 \mathrm{~g} / \mathrm{dl}$ increased progressively with the number of chemotherapy cycles in patients with lymphoma (Coiffier et al 2001).

When recombinant human erythropoietin (rHuEpo) became available around 1986, its use was first limited to patients with renal anemia. There were pilot studies in cancer from 1993 onwards, but it was not until about 10 years after rHuEpo had 


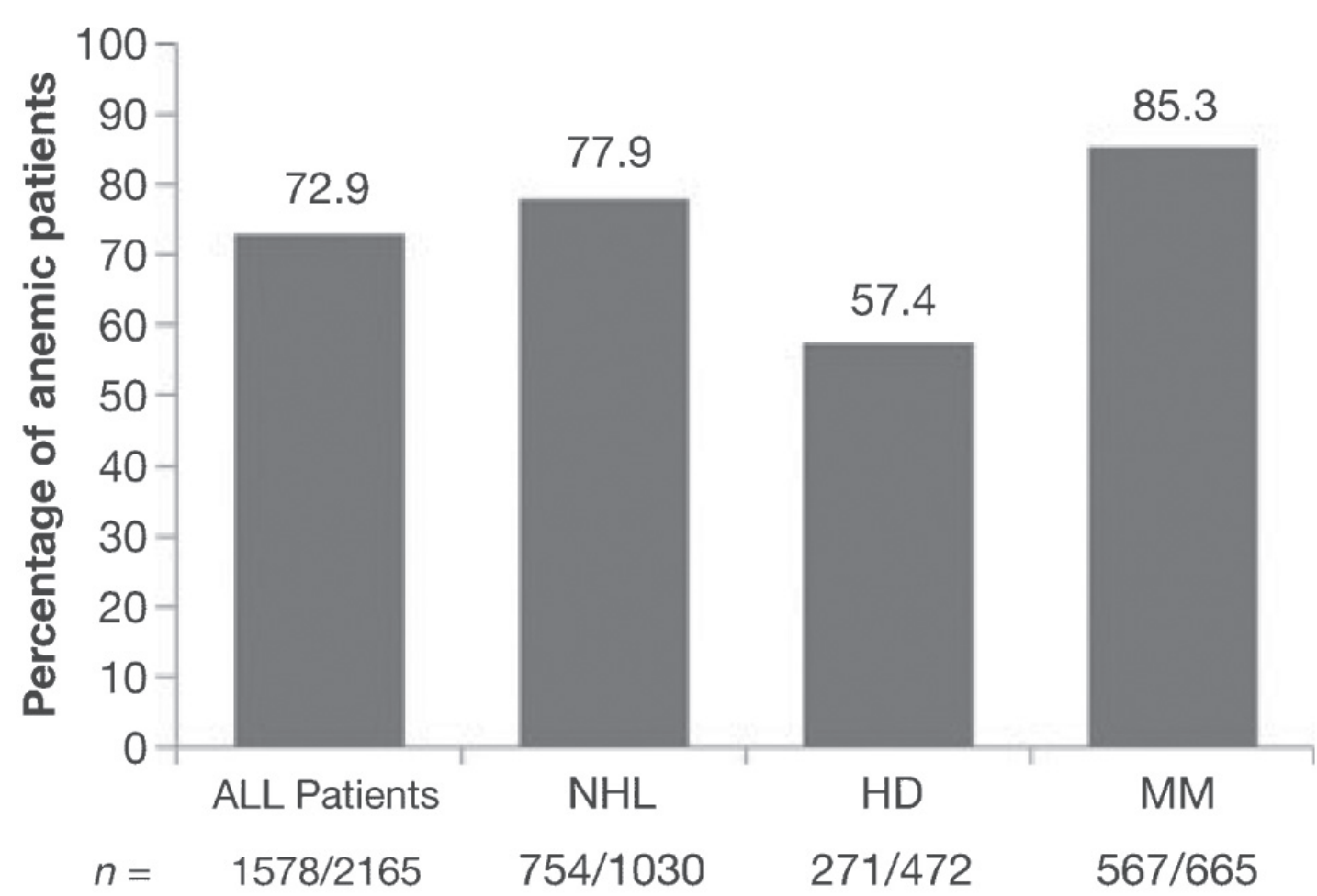

Figure I Frequency of anemia in 2165 lymphoma and myeloma patients during a 6 month follow-up period in the ECAS study. Copyright @ 2006 . Reproduced with permission from Birgegård G, Gascón P, Ludwig H. 2006. Evaluation of anemia in patients with multiple myeloma and lymphoma: findings of the European Cancer Anemia Survey. Eur J Haematol, 77:378-86.

become an established treatment form in renal anemia that larger studies in cancer anemia were undertaken. In recent years, the importance of the $\mathrm{Hb}$ level for physical capacity and quality of life (QoL) has been extensively investigated, and as a result the use of erythropoiesis-stimulating agents (ESA) in cancer patients has increased.

\section{Pathophysiology of cancer anemia}

Normal erythropoiesis is a dynamic and complex process that maintains the $\mathrm{Hb}$ level within a relatively narrow range under changing physiologic conditions (Mercadente et al 2000; Birgegård et al 2005). The homeostatic balance is upheld via a sensitive system where hypoxia induces transcription of erythropoietin (Epo), produced by the kidneys. The Epo response depends on the hypoxia signal, not on the absolute number of erythrocytes in the blood, and therefore compensating mechanisms may modify the Epo expression. The cardiovascular and respiratory response, the increase in 2,3BPG in the red cells, leading to a right-shift of the oxygen dissociation curve, increasing oxygen unloading, and lowering of the $\mathrm{pH}$ in the capillaries of vital organs, also enhancing oxygen delivery, all seek to compensate for a lowering of the oxygen carrying capacity. The efficacy of these compensatory mechanisms may vary between individuals, and this explains the variability in the expression of symptoms as well as the subjective experience of anemia in individual patients.

The anemia of cancer patients is a form of the anemia of chronic disease (ACD), which is a multifactorial condition, to a large extent caused by production of cytokines with a detrimental effect on the production of red cells (Figure 2). These cytokines are both inflammatory and paramalignant, and they exert their effect both on the level of Epo production (Miller et al 1990; Faquin et al 1992) and the proliferation and differentiation in the erythroblast compartment of the bone marrow (Mercadente et al 2000; Nowrousian 2002). Furthermore, there are important effects on the iron metabolism.

Inflammatory cytokines induce the expression of the most important iron distribution regulator hepcidin, which in turn down-regulates and increases degradation of ferroportin, the sole exporter of iron from within the cells to the plasma compartment (Roy and Andrews 2005; Nemeth and Ganz 2006). This leads to a decreased transport of iron across the gut brush border as well as from the macrophages to the plasma. Most of the iron released to plasma from macrophages comes from the break-down of senescent red cells. When this release of macrophage iron is decreased, plasma transferrin saturation is lowered, and the erythroblasts in the bone marrow are starved for iron. Simultaneously, iron is accumulated in the macrophages, explaining why these patients have a normal 


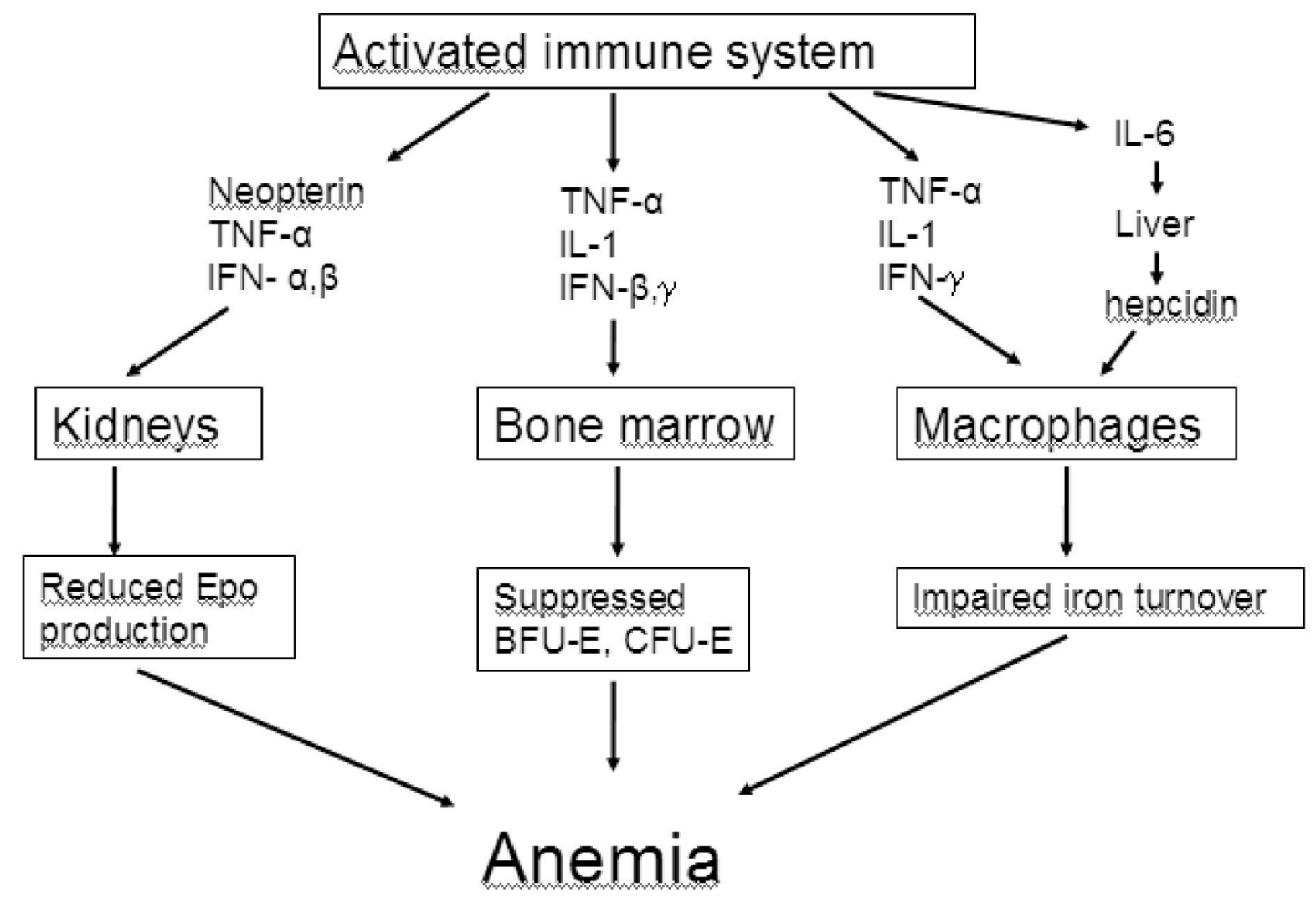

Figure 2 Pathophysiology of cancer anemia.

Abbreviations: IFN, interferon; IL, interleukin;TNF, tumor necrosis factor.

or even elevated s-ferritin level. The normal turnover of iron from the macrophages to the bone marrow erythroblasts comprises about $30 \mathrm{mg}$ of iron or 15-20 times the daily absorption, indicating how important this reutilisation of iron is in comparison with the daily uptake.

This hepcidin-induced disturbance of iron turnover has been named functional iron deficiency (FID) and seems to be a major problem in cancer anemia (Cavill et al 2006; Hedenus et al 2007). The problems with iron support of erythropoiesis in ACD become even more prominent during treatment with ESA, since it effectively hinders a response in many patients. Furthermore, if ESA treatment successfully stimulates red cell proliferation, more iron is needed. A doubling of the reticulocyte count is often achieved, and this also means a doubling of the iron need, or about $60 \mathrm{mg}$ of iron per day transported through plasma. Under other conditions, like in B12 deficiency anemia treatment, this is no problem, but in ACD the hepcidin-ferroportin interaction reduces the possibility for iron provision to the bone marrow.

Other contributing factors to the anemia of chronic disease are shortened red cell life span, nutritional deficiencies, and renal dysfunction.

\section{Anemia treatment in cancer}

As shown above, anemia is a common problem in cancer. The seriousness of the problem is a matter of controversy. Traditionally, a mild anemia has not been considered a condition worth treating, and even recently, the ECAS study showed that less than $50 \%$ of anemic patients had any anemia treatment (Ludwig et al 2004). One of the reasons for this controversy is probably that the tolerance for anemia is so variable; some patients have no symptoms and no decline in functional capacity even in moderate anemia. From this it has been tempting to conclude that symptoms in anemic patients are not due to the anemia itself.

However, a large number of studies, not least in nephrology patients, have shown that correcting anemia has led to improvements in physical capacity, cognitive function, and QoL. Meta-analysis of treatment studies in mixed cancer populations have shown not only that ESA treatment reduces transfusions and increases Hb levels (Seidenfeld et al 2001), but also has a positive effect on QoL (Glaspy et al 1997; Demetri et al 1998; Crawford et al 2002; Gabrilove et al 2001; Littlewood et al 2001; Quirt et al 2001; Cella 2002; Fallowfield et al 2002; Cella et al 2003a; Vansteenkiste and 
Wauters 2005; Witzig et al 2005). This was also related to fatigue studies in the general population, showing that the fatigue of cancer patients was far from "normal" (Cella et al 2003b). I nterestingly, a direct correlation between the magnitude of the $\mathrm{Hb}$ increase and the improvement of QoL was shown by Crawford and colleagues (2002). In an analysis of the studies by Glaspy, Demetri, and Gabrilove (a total of more than 6000 patients) an incremental analysis showed a correlation between $\mathrm{Hb}$ level and QoL score over the range of $8-14 \mathrm{~g} / \mathrm{dl}$, and the greatest improvement in QoL scores, for every $1 \mathrm{~g} / \mathrm{dl} \mathrm{Hb}$ increase, was seen in the range of 11 to $12 \mathrm{~g} / \mathrm{dl}$ (Crawford et al 2002).

Fatigue in cancer patients is multifactorial, and needs to be addressed with a wider perspective than just that of anemia. However, an important role for anemia in the fatigue of cancer patients has been found (Cella 2002).

These results have changed the attitudes to mild to moderate anemia, and recent guidelines for ESA treatment indicate a role for ESA in cancer anemia even in mild to moderate anemia (see below).

A further analysis of ESA treatment in solid tumor patients is outside the scope of this review. Recently, two studies have raised concerns that rHuEpo may adversely affect the survival and tumor progression of cancer patients (Henke et al 2003; Leyland-Jones et al 2005). In those trials, decreased survival and increased tumor progression were found in patients who received rHuEpo compared with patients who received placebo. Both studies have been criticized. Both used a $\mathrm{Hb}$ treatment goal above the recommended. In the breast cancer study, which included patients with very advanced disease, the death rate was high, and the difference was shown within the first three months, making it less likely that the rHuEpo treatment was the reason for the difference. In the head- and neck cancer study, there was an imbalance between the treatment arms with regard to smoking and the tumour status after operation, and in the patients actually treated according to protocol there was no significant difference. Nevertheless, caution has been recommended, especially during radiotherapy of solid tumours, and a number of hypotheses have been proposed to explain the potential role of ESAs, among them Epo receptor (EpoR) tumor stimulation, tumor neovascularization, and enhanced tumor oxygenation. A fundamental problem in the research on EpoRs is that the antibodies generally used are polyclonal, and detect other proteins as well. Therefore, it has been shown that anti-Epo receptor antibodies do not predict EpoR expression. EpoRs have been found in a variety of normal tissues at considerably lower expression levels than in bone marrow, and the levels found on tumors do not differ from normal tissue (Elliott et al 2006). In the in vitro tumor cell stimulation studies, suprapharmacological doses of rHuEpo have been used. So far, when this review is written, no in vivo animal tumor study has shown that treatment with ESAs alone enhances tumor progression or reduces survival. It is unfortunate that survival was not an end-point in the many previous ESA studies, but hopefully ongoing, well-controlled clinical trials will clarify the safety issue of ESA use in cancer.

\section{Anemia treatment in lymphoproliferative disease}

The anemia problem in lymphoma and multiple myeloma is greater than in many other cancer forms (Birgegård et al 2006). As cited above, the ECAS study showed that $73 \%$ of lymphoma and myeloma patients had a $\mathrm{Hb}<12 \mathrm{~g} / \mathrm{dl}$ at any time during a 6 month follow-up period, and during CHOP treatment $79 \%$ of the patients developed mild to moderate anemia and 49\% WHO grade 3-4 anemia in a study by Groopman and Itri (1999).

In the ECAS study, a significant correlation was found $(p<0.001)$ between WHO performance score and mean $\mathrm{Hb}$ at enrolment (Ludwig et al 2004). The lymphoma and myeloma population is generally old and comorbidity is common, also compromising the functional capacity and mental reserves of the patients.

\section{Efficacy of ESA treatment in lymphoma and myeloma patients}

Myeloma and lymphoma patients were included in a large number of ESA treatment studies with mixed cancer populations, both nonrandomized and randomized. Lately, a number of randomized, controlled studies have also been performed in myeloma and lymphoma patients. Response to ESA therapy has generally been defined as an increase in $\mathrm{Hb}$ of $>2 \mathrm{~g} / \mathrm{dl}$, and response rates vary between investigations, depending on the profile of the study populations. A typical ESA study in myeloma and lymphoma patients would have a response rate of $50 \%-70 \%$. Lower figures would be expected in trials with many patients with advanced disease and very low baseline $\mathrm{Hb}$, and better responses in patients with a higher baseline $\mathrm{Hb}$. This is supported by data from a randomized, placebo-controlled study, where response was seen in $68.5 \%$ of patients with a baseline $\mathrm{Hb} \leq 10.5 \mathrm{~g} / \mathrm{dl}$ compared with $80.5 \%$ in the patients with baseline $\mathrm{Hb} \geq 10.5 \mathrm{~g} / \mathrm{dl}$ (Dammacco et al 2001).

The efficacy of ESA treatment in increasing $\mathrm{Hb}$ levels was shown in a randomized, placebo-controlled study in 
nonmyeloid malignancies, where the responses rates were $67 \%$ vs $27 \%$ in the epoetin beta and placebo groups, respectively ( $p<0.0001$ ) (Osterborg et al 2002). Three large open-label, nonrandomized, community-based studies with more than 2000 patients each (Glaspy et al 1997; Gabrilove et al 2001), all showed a $\mathrm{Hb}$ increase of 1.8-2.0 g/dl from base-line, and this magnitude of the $\mathrm{Hb}$ increase has been common in later, randomized studies as well (Dammacco et al 2001; Hedenus et al 2003). The treatment time has generally been 12-16 weeks, enough to catch even late responders but of course too short for evaluation of impact on survival. There is almost no information about long-term ESA treatment in myeloma and lymphoma in randomized studies. The reasons for this are many: there are ethical considerations against keeping patients in a long-term control arm anemic, and there is difficulty in collecting large patient groups of long-term survivors who still are anemic. However, there is a large experience among clinicians of patients with long-term responses in palliative treatment of myeloma and lymphoma.

Due to the cost of ESA treatment, there has been reluctance among clinicians to prescribe these drugs, especially in situations where the anemia can be expected not to last for a long time. If the patient is not anemic at the start of tumor therapy, anemia may ensue a month or more into a 3-month long period of chemotherapy like CHOP-14 $\times$ VI, and since response to ESA treatment is mostly seen only after about 4-6 weeks, the time that the patient benefits may be too short to warrant the cost. It may be argued that the anemia is often more long-lasting than the treatment and that early intervention may make anemia avoidable.

\section{Effectiveness of ESA treatment in lymphoma and myeloma}

It is a matter of controversy whether an increase in $\mathrm{Hb}$ levels is a goal in itself in these patients. Anemia affects virtually all organs systems in the body (Ludwig and Strasser 2001), and it stands to reason that cancer patients would benefit from correcting anemia. However, ESA are expensive drugs, and doctors as well as funding authorities have expectations of objectively proven gains in order to support treatment.

Originally, the focus was on the avoidance of red cell transfusions, and in all studies it was seen that ESA treatment could reduce the number of transfusions. However, the effectiveness was questioned on the grounds that as many as four ESA-treated patients were needed to make one patient transfusion-independent (Seidenfeld et al 2002).

The strong correlation between prognosis and $\mathrm{Hb}$ levels at diagnosis makes it tempting to hypothesise that treatment of anemia would improve survival. In a small study in chronic lymphocytic leukemia (CLL) patients $(n=33)$, maintenance therapy with rHuEpo showed a direct and strong correlation to a sustained response to tumor therapy: all patients on rHuEpo maintenance remained responsive, whereas all patients without $\mathrm{rHuEpo}$ maintenance relapsed (Siakantaris et al 2000). The same group of investigators later showed that $15 / 20$ CLL patients could be down-staged in the Rai staging from stage III after response to Epo therapy. Chemotherapy was avoided or postponed in responders (Pangalis et al 2002). Unexplained myeloma regression in patients and antimyeloma effects in murine systems have been shown by one group (Mittelman et al 1997, 2001, 2004). However, in spite of these reports, meta-analyses fail to show an improved survival or tumor control in ESA-treated patients in randomized studies (Bohlius et al 2006a), and no larger single study has shown a significant survival advantage. Unlike in solid tumors, there is no indication of a negative effect in myeloma or lymphoma.

Since survival benefit has not been proven, other objective benefits must be shown to justify treatment. Therefore, the most obvious effect of increasing $\mathrm{Hb}$ levels from a clinical perspective, the improvement of QoL, has come into focus.

\section{Quality of life studies}

QoL has been studied in a large number of ESA treatment studies in cancer and the results have constantly been positive for ESA treatment. This is true for solid tumors as well as myeloma and lymphoma and for patients with or without chemotherapy. The most widely used QoL measurement is the Functional Assessment of Cancer Therapy (FACT) instrument with its anemia subscale FACT-An, published and validated by Cella and others (Cella 1997; Yellen et al 1997). A prospective, randomized, placebo-controlled study with over 300 myeloma and lymphoma patients showed major improvements in FACT-An as well as Linear Analogue Scales (LASA) for the patients who had a $\mathrm{Hb}$ improvement from ESA treatment (Littlewood et al 2001), and this univariate analysis was later supported by a multiple linear regression analysis of the same material, accounting for disease progression and other possible confounding factors (Fallowfield et al 2002). Hedenus and colleagues (2003) found a statistically significant relationship $(\mathrm{p}<0.001)$ between change in $\mathrm{Hb}$ and change in FACT-An scores and also showed that patients with a lower base-line FACT-An score reported the largest improvements. A correlation between the magnitude of the $\mathrm{Hb}$ change and the QoL gain was earlier found in the large 
nonrandomized study by Gabrilove and colleagues (2001) (Figure 3). Dammacco and colleagues (2001) showed, apart from an improvement in QoL in the ESA treatment arm, also a significantly better performance score in the treated patients. In a study of 349 myeloma and lymphoma patients, Österborg and colleagues (2002) confirmed a QoL gain in the ESA-treated patients and a correlation between $\mathrm{Hb}$ increase and QoL improvement.

From all these studies, and others, it may be concluded that improvement in QoL is strongly correlated to $\mathrm{Hb}$ increase, a correlation that gives strong support for the notion that anemia is an important factor in the symptoms and the well-being of cancer patients.

Unfortunately, there is a lack of data concerning objective measures of effects on physical performance, cognitive functions, cardiovascular effects, etc., in the literature on treatment of cancer anemia. This is in contrast to the area of renal anemia, where such studies have given support to the importance of correction of anemia.

\section{Indications for ESA treatment}

Before initiating ESA therapy, the clinician would like to have reliable indicators of the chance for a positive treatment response. Attempts have been made to identify patients with a small chance for response in order to avoid fruitless treatment attempts. The baseline s-Epo level has been identified as a predictor on the group level (Henry et al 1995; Cazzola et al 1996; Macdougall et al 1996; Macdougall 1998). This has been challenged in recent studies, especially in myeloma and lymphoma patients (Katodritou et al 2004). Also, in the individual patient a cut-off level identified as an optimal predictor for the group has little value. With a s-Epo above $100 \mathrm{U} / \mathrm{L}$, which has often been found to be a group response predictor, still a considerable percentage of cancer patients respond to ESA therapy. Furthermore, cancer patients have a blunted Epo response to anemia (Miller et al 1990), a vast majority of them showing s-Epo levels below $200 \mathrm{u} / \mathrm{L}$ (Henry et al 1995). Therefore, s-Epo measurement before treatment start has limited value.

Several guidelines for ESA treatment have been published (Bokemeyer et al 2004; Djulbegovic 2005). Since new studies tend to change the recommendations, the value of guidelines is closely connected to the year of publication. The most recent are the updated EORTC guidelines, published last year (Bokemeyer et al 2007). Forty-three new publications and 78 relevant abstracts were added since the last update 3 years earlier. In the new EORTC guidelines, which states the evidence grade for each recommendation, initiation of therapy is recommended at a $\mathrm{Hb}$ level of 9-11 g/dl, based on anemia-related symptoms.

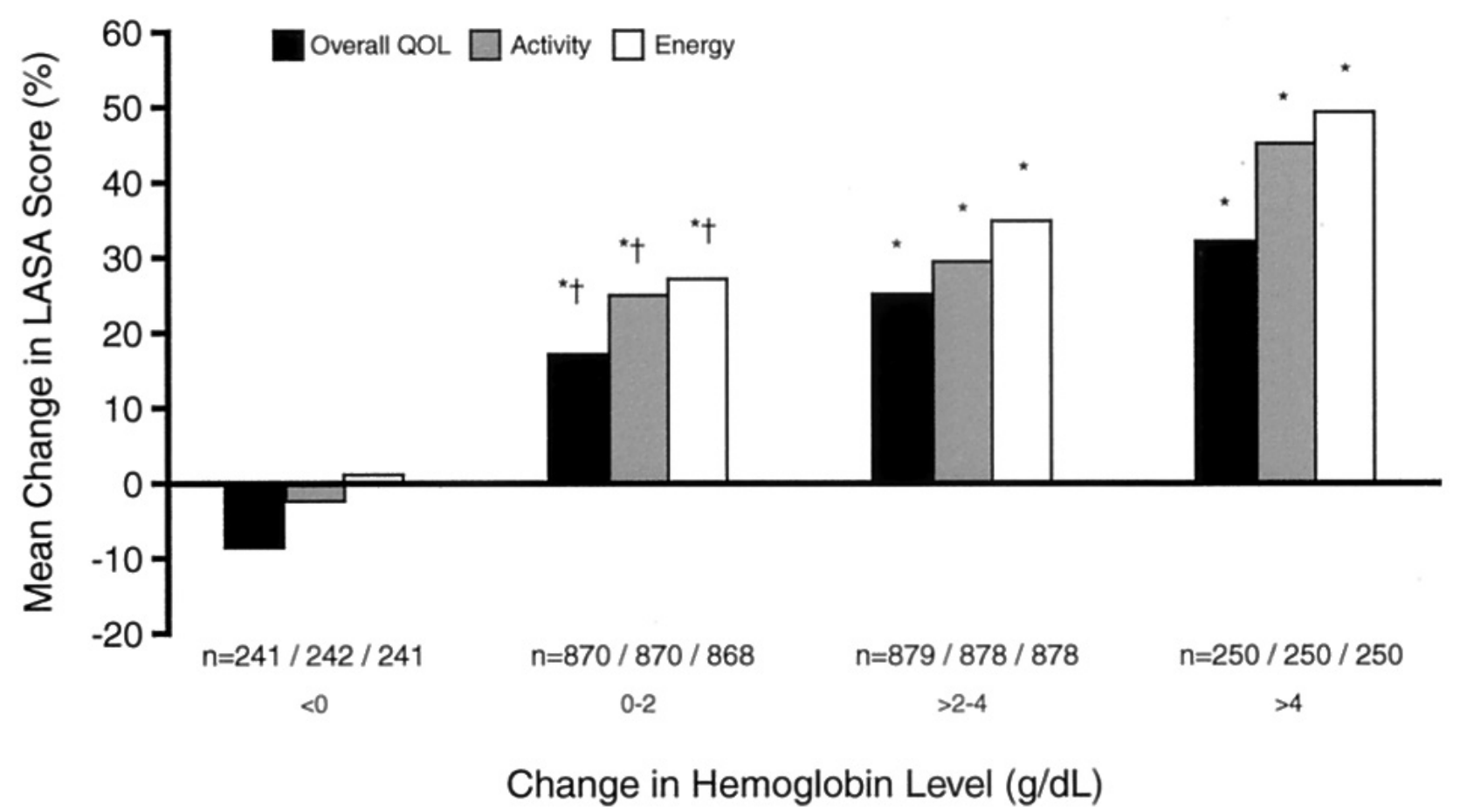

Figure 3 The relationship between increase in $\mathrm{Hb}$ and change in LASA QoL scores. The more Hb levels increased, the greater was the improvement in QoL scores. Copyright (C) 200I. Reprinted with permission from the American Society of Clinical Oncology. Gabrilove JL, Cleeland CS, Livingston RB, et al 200I. Clinical evaluation of once-weekly dosing of epoetin alfa in chemotherapy patients: improvements in hemoglobin and quality of life are similar to three-times-weekly dosing.J Clin Oncol, I9:2875-82. 
Table I Condensed EORTC 2007 guidelines for erythropoiesisstimulating agents treatment

\begin{tabular}{ll}
\hline $\mathrm{Hb}$ initiation level & $9-\mathrm{II} \mathrm{g} / \mathrm{dl}$ \\
Early intervention, & Yes, with falling $\mathrm{Hb}$ and further \\
$\mathrm{Hb}<\mathrm{I} 2$ & chemotherapy planned \\
Prophylactic treatment & No \\
Additional oral iron & Yes \\
Additional lv iron & Treatment as younger \\
Elderly patients & No \\
Dose escalation & Epo alfa and beta QW, darbepoetin \\
Dosing & QW or Q 3W \\
& I2-I3 g/dl \\
Target Hb level & QoL improvement and transfusion \\
Goal of therapy & prevention \\
Higher starting dose & No
\end{tabular}

Abbreviation: QoL, quality of life.

However, treatment may be started even at a $\mathrm{Hb} \leq 12 \mathrm{~g} / \mathrm{dl}$ to prevent further decline in $\mathrm{Hb}$ in patients with falling $\mathrm{Hb}$ levels and a planned further cytostatic treatment with a high risk of worsening anemia. The target $\mathrm{Hb}$ level is given as $12-13 \mathrm{~g} / \mathrm{dl}$, and the two major goals of treatment should be improvement of QoL and avoidance of red blood cell transfusions (Table 1).

In this context it should be pointed out that a reduction of previous levels of physical and social function should be defined as a symptom. Patients with anemia tend to reduce their activities in order to avoid open symptoms of anemia and this reduction of capacity may easily go unnoticed.

A recent study by Straus and colleagues (2006) is of special interest with regard to the initiation of ESA therapy. Almost 300 patients with lymphproliferative disease were randomized to early $(\mathrm{Hb} \leq 12 \mathrm{~g} / \mathrm{dl})$ or late $(<9 \mathrm{~g} / \mathrm{dl})$ treatment start. All had a transferrin saturation $>20 \%$ and s-ferritin $>50 \mathrm{ug} / \mathrm{L}$. The treatment goal was $\mathrm{Hb} 13-15 \mathrm{~g} / \mathrm{dl}$. The Hb level increased in the "early" treatment group and decreased in the "late" group ( $p<0.0001)$. More early patients achieved a hematological response, $70 \%$ vs $25 \%$, giving support to conclusions in previous studies, where patients with a more advanced anemia responded more poorly. QoL was investigated with FACT-An and LASA, and there was a significant difference between groups with both instruments. The mean change in the total FACT-An favored early patients by 8.2 points $(\mathrm{p}=0.003)$, and early patients had significantly more improvement in all three LASA domain scores for daily activity $(p=0.008)$, energy $(\mathrm{p}=0.007)$, and important activity $(\mathrm{p}=0.045)$. Again, the correlation between treatment-related changes in $\mathrm{Hb}$ level and QoL effects was demonstrated $(\mathrm{p}<0.05)$.
This study gives support for the selection of patients for treatment who start to decline in $\mathrm{Hb}$ level early in a series of cytostatic treatments. It has previously been shown that a low-normal $\mathrm{Hb}$ level at the start of cytostatic treatment is the strongest predictive factor for later anemia (Ludwig et al 2004), both in cancer in general and in myeloma and lymphoma (Birgegård et al 2006). A recent analysis of the benefit of early anemia treatment gives further support to this strategy (Lyman and Glaspy 2006).

Cancer patients survive longer in modern medicine, and palliative treatment may extend over long periods of time. Therefore, ESA treatment is not only indicated in patients given curative chemotherapy. It may be argued that upholding a good QoL through anemia correction is even more important in patients who have indolent or progressive disease and a life expectancy of more than a few months. Transfusion therapy usually does not aim at keeping the $\mathrm{Hb}$ level higher than about $10 \mathrm{~g} / \mathrm{dl}$, and recent studies show important gains in QoL when $\mathrm{Hb}$ is increased from this level to above12 $\mathrm{g} / \mathrm{dl}$.

\section{A new concept: Improved response with IV iron addition}

There are two reasons why addition of iron could be beneficial for response to ESA. First of all, some patients have small iron stores that will be rapidly exhausted if erythropoiesis is stimulated. A plasma ferritin below 100 indicates that iron stores are too small to sustain a new production of red cells from a moderate anemia to normal $\mathrm{Hb}$ levels. Secondly, cancer patients often have FID, a condition where the turnover of iron from senescent red cells is disturbed: iron is locked inside macrophages and the transport of iron to the erythroblasts is reduced, causing iron starvation of the erythroblasts (see above). FID therefore is characterized by a normal or elevated plasma ferritin, indicating presence or even elevation of iron stores, and low transferrin saturation. Apart from these simple measurements, other analyses may be used. In all situations where the erythroblasts need more iron more transferrin receptors (TfR) will be expressed on the surface of the cells, and the level of soluble sTfR increases. Transferrin saturation (Tsat) may be said to represent the functional iron compartment, whereas s-ferritin represents the storage compartment. A combination of serum ferritin and sTfR, the so called sTfR-F index, is a very reliable indicator of depleted iron stores (Punnonen et al 1997).

\section{Diagnosis of functional iron deficiency}

There is a need for identification of patients with FID before treatment, since this condition is a hinder for response to ESA. 
In renal anemia, this problem is well known and described, and it has been shown that the measurement of the percentage of hypochromic red cells in the blood (\% HYPO) and the hemoglobin content of reticulocytes $(\mathrm{CHr})$ are reliable indicators of FID (Fishbane et al 1997; Macdougall 1998). In cancer anemia, a combination of \% HYPO, TfR, and $\mathrm{CHr}$ have been used to create a diagnostic plot which effectively identifies FID (Thomas et al 2006). Recently, this has been simplified to include \% HYPO and sTfR index and most recently \% HYPO alone (Katodritou et al 2007). Unfortunately, the most commonly used automated analysis systems like Sysmex or Coulter can not measure \% HYPO or $\mathrm{CHr}$, and clinicians are therefore forced to rely on the less reliable sTfR alone or, in many cases, Tsat in combination with s-ferritin. The sensitivity and positive predictive value of Tsat alone is rather low (Katodritou et al 2007). As a practical and pragmatic rule for clinicians without access to \% HYPO, CHr, or sTfR, the combination of normal or high ferritin and low Tsat $(<20 \%)$ is a strong indication of functional iron deficiency. Lately, a new marker that can be analyzed with the Sysmex system, the RET-Y, has been investigated. The RET-Y is the mean value of the forward-scattered light histogram within the reticulocyte population. A close correlation with $\mathrm{CHr}$ was found in 474 patients (Franck et al 2004). The use of this variable in a cancer population has yet to be defined.

The importance of changes in iron turnover in cancer anemia was not clear when many of the ESA studies were planned, and often information about iron supplementation is missing. Generally, iron deficiency, defined as a low serum ferritin level, has been an exclusion criterion. However, investigation of bone marrow iron has rarely been performed, and diagnosis of FID has not been made. Iron supplementation, when defined, has mostly been given orally.

\section{Results of IV iron addition in cancer anemia}

In renal anemia, the concept of FID and its treatment have been well known. The superiority of IV over oral iron supplementation has been shown, and a ESA-saving effect has been found in randomized, controlled studies already in the mid-90's (Fishbane et al 1995; Macdougall et al 1996; Auerbach et al 2004). In cancer anemia, the first randomized study showing an increased $\mathrm{Hb}$ response with IV iron was published in 2004 (Auerbach et al 2004). This was a randomized study in 157 patients receiving chemotherapy treatment and $\mathrm{rHuEpo}$ 40,000 U per week and compared the addition of oral or IV iron dextran to no iron. A significant difference in response rates was found, $68 \%$ for IV iron vs. 36\% for oral iron and $25 \%$ for no iron $(\mathrm{p}<0.01)$. Eligible for the study were patients with
$\mathrm{Hb}<10.5 \mathrm{~g} / \mathrm{dl}$ and $\mathrm{s}-\mathrm{ferritin} \leq 450 \mathrm{pmol} / \mathrm{L}$ or $\leq 675 \mathrm{pmol} / \mathrm{L}$ and Tsat $\leq 19 \%$. Bone marrow iron staining was not performed. The study has been questioned because of the unusually low response rates in the no-iron and oral-iron groups, which may have been caused by the short treatment duration, 6 weeks, and by the presence of true iron deficiency.

In another randomized, open-label study, 187 nonmyeloid patients with chemotherapy-related anemia were scheduled to receive $\mathrm{rHuEpo} 40,000 \mathrm{U} /$ week, one group receiving $125 \mathrm{mg}$ of ferric gluconate IV once weekly, another group $325 \mathrm{mg}$ or oral ferrous sulphate three times daily and a third group no iron supplementation (Henry et al 2007). The inclusion criteria differed from the previous study: $\mathrm{Hb}<11 \mathrm{~g} / \mathrm{dl}$, $\mathrm{s}$-ferritin $\geq 100 \mathrm{ng} / \mathrm{ml}$ or Tsat $\geq 15 \%$. In this way, true iron deficiency was probably to a large extent avoided, although no bone marrow iron staining was performed. However, patients with FID and s-ferritin $>100 \mathrm{ng} / \mathrm{ml}$ and Tsat $<15 \%$ may have been excluded. The treatment time was short also in this study, 8 weeks. Of the 187 patients recruited, 154 completed the study and only 129 were evaluable for efficacy, mainly due to early transfusions or discontinuations. The results showed significant differences between the Iv iron group and the other two groups in $\mathrm{Hb}$ increase, $2.4 \mathrm{~g} / \mathrm{dl}$, $1.6 \mathrm{~g} / \mathrm{dl}$, and $1.5 \mathrm{~g} / \mathrm{dl}$, respectively ( $\mathrm{p}=0.0044$ for IV vs. no iron), in response rates, $73 \%, 45 \%$, and $41 \%$ respectively ( $p=0.029$ for IV vs. no iron). Interestingly, the subgroup with the lowest Tsat, below $20 \%$, had a response rate of $81 \%$ in the IV iron group compared with $37 \%$ in the oral iron group and $27 \%$ in the no-iron group. It may be assumed that most of the patients with low Tsat had FID, even though some true iron deficiency patients may have been included. Patients with Tsat $>20 \%$ had a lower response rate to IV iron than those with Tsat $<20 \%$ (68 vs $81 \%$ ). This underlines the importance of the iron need, indicated by a low Tsat.

The third and most recent study (Hedenus et al 2007) randomized 67 lymphoproliferative patients without chemotherapy, with $\mathrm{Hb}$ 9-11 g/dl and proven iron stores, shown by a positive bone marrow Prussian blue staining to receive rHuEpo 30,000 U once weekly plus or minus IV iron sucrose $100 \mathrm{mg}$ weekly during week 1-6, then every second week during week $8-14$. The treatment time was 16 weeks. The study was powered to detect a mean $\mathrm{Hb}$ increase difference of $1 \mathrm{~g} / \mathrm{dl}$. There was a significant difference between the IV iron group and the no-iron group with regard to response rate $(\mathrm{Hb}$ increase $>2 \mathrm{~g} / \mathrm{dl}), 93 \%$ vs. $53 \%(\mathrm{p}=0.001)$ (Figure 4$)$. The time to response was half as long and the $\mathrm{Hb}$ increase was greater (2.91 vs. $1.5 \mathrm{~g} / \mathrm{dl}, \mathrm{p}=0.0001)$. The figures for per protocol- and intention-to-treat populations were similar. 


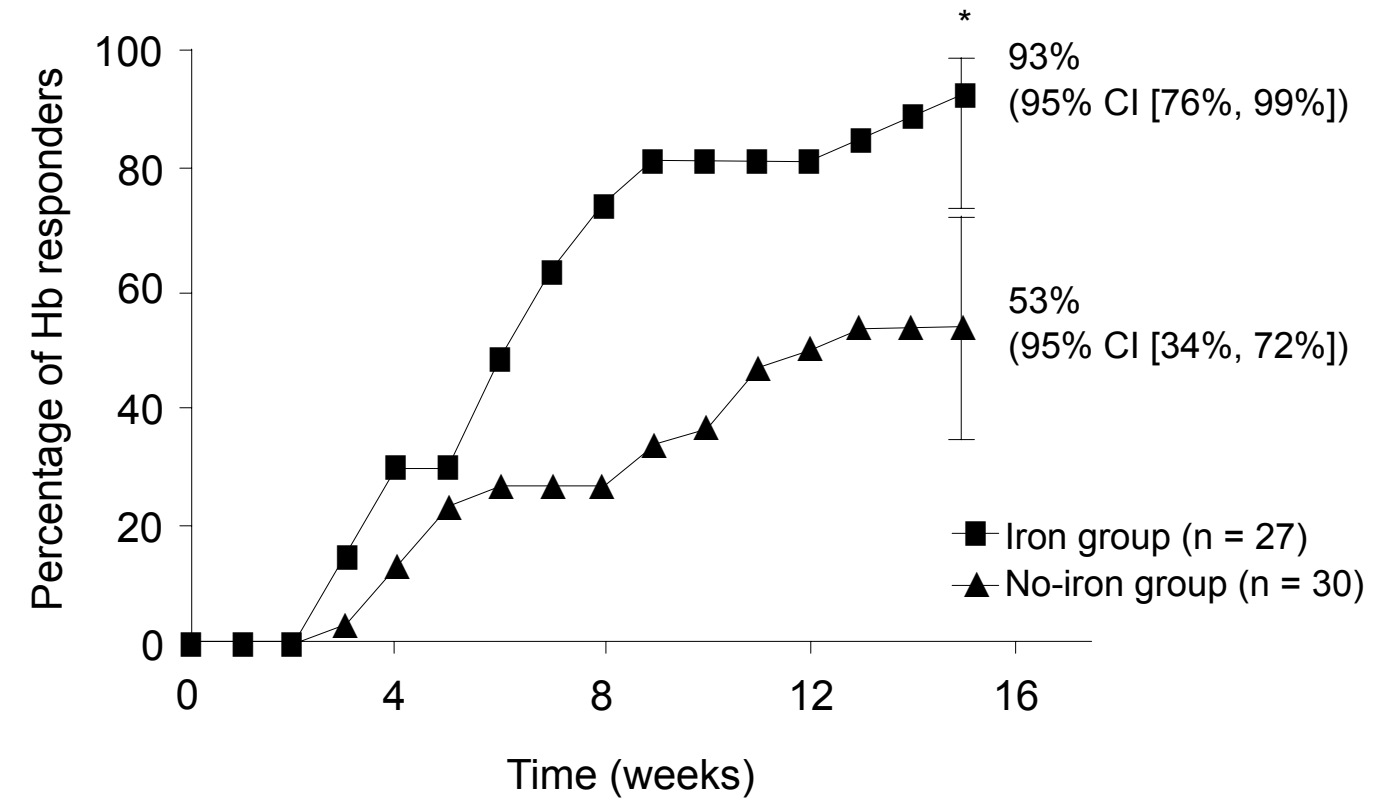

\#Increase in $\mathrm{Hb}$ of $\geq 2 \mathrm{~g} / \mathrm{d}$ during the study without transfusion. *Significant difference $(p=0.0012)$ between treatment erms.

Figure 4 Hemoglobin response rates were significantly higher in the group with additional IV iron during ESA treatment, $93 \%$ vs $53 \%$, and the response (Hb increase $>2$ g/dl) was twice as quick in this group. Copyright (C) 2007. Reproduced from Hedenus M, Birgegård G, Näsman P, et al 2007.Addition of intravenous iron to epoetin beta increases hemoglobin response and decreases epoetin dose requirement in anemic patients with lymphoproliferative malignancies: a randomized multicenter study. Leukemia, $21: 627-32$.

Since true iron deficiency was excluded by bone marrow iron staining in this study, the $39 \%$ of the whole population with Tsat $<20 \%$ may be regarded as FID, underlining the importance of this condition in cancer patients. All patients with Tsat $<20 \%$ in the IV iron group responded with a $\mathrm{Hb}$ increase $>2 \mathrm{~g} / \mathrm{dl}$. The difference in treatment results between the IV iron and no-iron groups was also due to the fact that some patients in the no-iron group had iron stores insufficient to support a $\mathrm{Hb}$ increase $>2 \mathrm{~g} / \mathrm{dl}$, showed by the fact that one third of these patients reached subnormal s-ferritin levels during the study and that the Tsat fell below $20 \%$ in all the patients in this group with a normal Tsat at base-line.

A reduction in rHuEpo need was seen in the IV iron group. At the end of the study, a 25\% difference (10000 U per week) in Epo dose was seen between the arms, and the mean total cumulative dose per patient was 511 vs. 626 thousand $U(p=0.051)$. The study was not powered to prove this difference, but the borderline significance is notable and supports results from the studies in renal anemia. The difference translates into a cost reduction of at least $\$ 100$ per week, depending on local list prices of ESA.

In conclusion, the improvement in efficacy of ESA treatment by addition of IV iron has now been convincingly shown both in renal and cancer anemia. The mechanism is known. Some questions remain unanswered, though. How many patients with FID would respond to monotherapy with IV iron without ESA? Would oral iron treatment suffice for patients with small iron stores (s-ferritin $<100 \mathrm{ug} / \mathrm{L}$ but within the reference range) and no sign of FID (Tsat $>20$, normal \% HYPO)?

\section{Are there risks with IV iron treatment?}

So far, no negative effects of iron addition have been found. However, no study has been powered or long enough for survival analysis. There is a fear that iron overload may ensue, especially in maintenance treatment, and that this would cause the formation of toxic oxygen radicals that could promote tumour growth or cause organ damage. There is room for some caution, therefore, and in further studies, survival needs to be an important endpoint. A reasonable mode of treatment is to monitor the s-ferritin levels, making certain that gross iron overload is avoided by reducing iron doses when s-ferritin increases over a certain limit. This means making sure that the patient uses the iron given for erythropoiesis. It is a matter of debate what the s-ferritin limit should be, and there is no data in the literature to support the 
choice. It must be remembered that s-ferritin often is elevated beyond the level reflecting the iron stores in cancer patients due to its property as an acute phase reactant, responding to inflammatory cytokines.

The EORTC guidelines were published before the last two studies of IV iron supplement in cancer anemia. Still, the guidelines state that there was grade B evidence to support improved response of ESA with addition of IV iron and that oral iron is ineffective in functional iron deficiency.

It is obvious that both functional iron deficiency and small iron stores are very important factors to consider when starting ESA treatment.

\section{Evaluation of treatment effects}

Randomized, placebo-controlled studies have convincingly shown the efficacy and effectiveness of ESA therapy for cancer anemia in general and for anemia in myeloma and lymphoma, for patients with or without chemotherapy. The effectiveness is now well established on a group level, and an individualised approach is necessary. All patients do not respond, and all patients who respond with an increase in $\mathrm{Hb}$ do not experience QoL gains. Therefore, a careful monitoring of individual patients is necessary with an evaluation of treatment effects, objectively and subjectively. In clinical practice, a part of the high cost of ESA treatment in total is probably a waste of drug in patients who are not taken off treatment in spite of non-responsiveness. An increase in $\mathrm{Hb}$ should be expected within 6 weeks. Unfortunately, there is no reliable early marker of response. Attempts have been made to combine markers like reticulocyte counts, transferrin receptor and s-ferritin in order to identify nonresponding patients within the first weeks of treatment (Cazzola et al 1996; Beguin 1998; Tonelli et al 2001), but none of these has been consistently reliable. A recent attempt to use the increase in beta-globin mRNA during the first two weeks of therapy is interesting but needs further investigation (Hagberg et al 2003; Birgegård et al 2007). Still, Hb increase after 4-6 weeks is the only reliable response indicator.

With regard to effectiveness in alleviating symptoms or improving functional capacity, the QoL instruments used in clinical trials, although showing good consistency and reliability, are generally too cumbersome to use with individual patients. Van Belle and colleagues (2005) have compared proposed diagnostic criteria with FACT-F and VAS for cancer-related fatigue. The internal consistency of the ICD-10 and the FACT-F, expressed as Crohnbach's alpha coefficient were 0.82 and 0.89 , respectively, in a material of 834 patients. The diagnostic tool ICD-10 and FACT-F correlated well for individual symptoms, except for items assessing memory performance and emotional problems. It was concluded that both instruments evaluate physical symptoms more reliably than psychological and emotional. The ICD-10 was recommended as a diagnostic tool, whereas the FACT-F scale also assesses the intensity of fatigue and is suitable for follow-up studies.

In comparison to the other two, the VAS (or LASA) tool is easy and rapid to complete and very intuitive for patients. On a visual analogue scale the patient indicates a value for three domains: energy level, activity level and general QoL. The VAS scale showed good agreement with the other two and is rapid, easy-to-use tool for longitudinal follow-up.

\section{Dosing and choice of ESA}

Attempts have been made to increase efficacy by using a higher dose of ESA during the first weeks of therapy (Hesketh et al 2004). Only one study has compared the standard fixed dose versus weight-based dose. In both cases, there is not enough evidence to support these strategies (Ludwig et al 2004).

The earlier standard dosing of three times weekly has now been largely abandoned since more than fifty studies have provided evidence for less frequent dosing, the most common being once weekly. Darbepoetin alfa, which was designed to have a longer half-life, has been shown to be effective with a Q3w dosing (Steensma et al 2006). In clinical practice, many have seen that patients with a good hematopoietic response to ESA may be maintained with Q2W or Q3W dosing of Epo $\alpha$ or $\beta$ as well (personal experience). The choice of ESA is not a matter of efficacy, and all ESA on the market are recommended in the published guidelines.

New compounds that are not Epo molecules, but are smaller peptides stimulating the Epo receptor are being investigated but are not yet on the market and are not included in this survey.

\section{Possible negative effects of ESA treatment in myeloma and lymphoma}

Cancer patients in general have an increased incidence of thrombosis, and a further increase has been found in ESAtreated patients. A meta-analysis with data from 9353 patients in 57 trials concluded that there was evidence that ESA significantly increased the risk of thrombosis or related complications ( $R R=1.67$; CI 1.35, 2.06) (Bohlius et al 2006b). The recommended treatment goal of $\mathrm{Hb} 12 \mathrm{~g} / \mathrm{dl}$ should be used in order to minimize this risk.

Pure red cell aplasia (PRCA) is a very rare complication of ESA treatment. Any protein injected into a human 
may cause antibody formation, and ESA are no exceptions. However, biologically active antibodies against erythrocyte precursors are rare, except during a period of a few years after 1998, when one of the ESA preparations due to a change in the manufacturing process gave rise to about 200 cases of PRCA world-wide (Casadevall et al 2002). Almost all cases had renal anemia, and after 2002, when the manufacturing process was changed again, the incidence of PRCA has returned to a very low level, again making it a rare problem.

The ongoing discussion about possible negative effects on survival and tumor control in solid tumors is of less relevance in hematological malignancies. There is no study indicating any negative effect. On the contrary, there are a few studies indicating a positive effect, and a recent meta-analysis (Bohlius et al 2006a) as well as a long term follow-up study (Osterborg et al 2005) fail to show any difference between ESA treatment and placebo. As discussed above, the presence of EpoRs on tumor cells is not surprising, since a wide variety of normal tissues also express the EpoR in the same amounts. In lymphoproliferative disease, no in vivo or in vitro data indicate any tumour stimulation.

\section{Conclusions}

ESA treatment of anemia in lymphproliferative disorders has been shown to be both efficacious and effective. Even if avoidance of red cell transfusions is an important treatment goal, the main reason for ESA treatment is improvement of QoL. Recent guidelines stress the latter goal and recommend treatment also in mild and moderate anemia, related to symptoms. The recent findings of the drug cost saving and the improvement of response rates with the addition of IV iron is an important step forward in optimizing ESA treatment.

\section{Disclosure}

The author has occasionally been involved in advisory boards for NeoRecormon ${ }^{\circledR}$, Darbepoetin ${ }^{\circledR}$, and Dynepo ${ }^{\circledR}$. There are no other conflicts of interest to report.

\section{References}

Auerbach M, Ballard H, Trout JR, et al. 2004. Intravenous iron optimizes the response to recombinant human erythropoietin in cancer patients with chemotherapy-related anemia: a multicenter, open-label, randomized trial. J Clin Oncol, 22:1301-7.

Barrett-Lee PJ, Ludwig H, Birgegård G, et al. 2006. Independent risk factors for anemia in cancer patients receiving chemotherapy: results from the European Cancer Anemia Survey. Oncology, 70:34-48.

Beguin Y. 1998. Prediction of response to treatment with recombinant human erythropoietin in anemia associated with cancer. Med Oncol, 15(Suppl 1):S38-46.
Birgegård G, Aapro MS, Bokemeyer C, et al. 2005. Cancer-related anemia: pathogenesis, prevalence and treatment. Oncology, 68(Suppl 1):3-11.

Birgegård G, Gascón P, Ludwig H. 2006. Evaluation of anemia in patients with multiple myeloma and lymphoma: findings of the European Cancer Anemia Survey. Eur J Haematol, 77:378-86.

Birgegård G, Dahl F, Glimelius B, et al. 2007. Evaluation of beta globin mRNA as an early marker of haemoglobin response to epoetin treatment. Med Oncol, 24:318-22.

Bohlius J, Wilson J, Seidenfeld J, et al. 2006a. Recombinant human erythropoietins and cancer patients: updated meta-analysis of 57 studies including 9353 patients. J Natl Cancer Inst, 98:708-14.

Bohlius J, Weingart O, Trelle S, et al. 2006b. Cancer-related anemia and recombinant human erythropoietin - an updated overview. Nat Clin Pract Oncol, 3:152-64.

Bokemeyer C, Aapro MS, Courdi A, et al. 2004. EORTC guidelines for the use of erythropoietic proteins in anaemic patients with cancer. Eur J Cancer, 40:2201-16.

Bokemeyer C, Aapro MS, Courdi A, et al. European Organisation for Research and Treatment of Cancer (EORTC) Taskforce for the Elderly. 2007. EORTC guidelines for the use of erythropoietic proteins in anaemic patients with cancer: 2006 update. Eur J Cancer, 43:258-70.

Casadevall N, Nataf J, Viron B, et al. 2002. Pure red cell aplasia and antierythropoietin antibodies in patients treated with recombinant erythropoietin. N Eng J Med, 346:469-75.

Cavill I, Auerbach M, Bailie GR, et al. 2006. Iron and the anemia of chronic disease. Curr Med Res Opin, 22:731-37.

Cazzola M, Ponchio L, Pedrotti C, et al. 1996. Prediction of response to recombinant human erythropoietin in anemia of malignancy. Hematologica, 81:434-41.

Cella D. 1997. The Functional Assessment of Cancer-Therapy Anemia (FACT-An) Scale: a new tool for the assessment of outcomes in cancer anemia and fatigue. Semin Hematol, 34:13-19.

Cella D. 2002. The effects of anemia and anemia treatment on the quality of life of people with cancer. Oncology (Williston Park), 16(9 Suppl 10):125-32.

Cella D, Dobrez D, Glaspy J. 2003a. Control of cancer-related anemia with erythropoietic agents: a review of evidence for improved quality of life and clinical outcomes. Ann Oncol, 14:511-19.

Cella D, Zagari MJ, Vandoros C, et al. 2003b. Epoetin alfa treatment results in clinically significant improvements in quality of life in anemic cancer patients when referenced to the general population. J Clin Oncol, 21:366-73.

Coiffier B, Guastalla JP, Pujade-Lauraine E, et al. Anemia Study Group. 2001. Predicting cancer-associated anemia in patients receiving nonplatinum chemotherapy. Eur J Cancer, 37:1617-23.

Crawford J, Cella D, Cleeland CS, et al. 2002. Relationship between changes in hemoglobin level and quality of life during chemotherapy in anemic cancer patients receiving epoetin alfa therapy. Cancer, 95:888-95.

Dammacco F, Castoldi G, Rödjer S. 2001. Efficacy of epoetin alfa in the treatment of anemia of multiple myeloma. $\mathrm{Br} J$ Haematol, 113:172-9.

Demetri GD, Kris M, Wade J, et al. 1998. Quality-of-life benefit in chemotherapy patients treated with epoetin alfa is independent of disease response or tumor type: results from a prospective community oncology study. Procrit Study Group. J Clin Oncol, 16:3412-25.

Djulbegovic B. 2005. Erythropoietin use in oncology: a summary of the evidence and practice guidelines comparing efforts of the Cochrane Review group and Blue Cross/Blue Shield to set up the ASCO/ASH guidelines. Best Pract Res Clin Haematol, 18:455-66.

Elliott S, Busse L, Bass MB. 2006. Anti-Epo receptor anitbodies do not predict Epo receptor expression. Blood, 107:1892-5.

Fallowfield L, Gagnon D, Zagari M, et al. for the Epoetin Alfa Study Group. 2002. Multivariate regression analyses of data from a randomised, double-blind, placebo-controlled study confirm quality of life benefit of epoetin alfa in patients receiving non-platinum chemotherapy. $\mathrm{Br} J$ Cancer, 87:1341-53. 
Faquin WC, Schneider TJ, Goldberg MA. 1992. Effect of inflammatory cytokines on hypoxia-induced erythropoietin production. Blood, 79:1987-94.

Fishbane S, Frei GL, Maesaka J. 1995. Reduction in recombinant human erythropoietin doses by the use of chronic intravenous iron supplementation. Am J Kidney Dis, 26:41-6.

Fishbane S, Galgano S, Langley RC Jr, et al. 1997. Reticulocyte hemoglobin content in the evaluation or iron status of hemodialysis patients. Kidney Int, 52:217-22.

Franck S, Linssen J, Messinger M, et al. 2004. Potential utility of Ret-Y in the diagnosis of iron restricted erythropoiesis. Clin Chem, 50:1240-2.

Gabrilove JL, Cleeland CS, Livingston RB, et al. 2001. Clinical evaluation of once-weekly dosing of epoetin alfa in chemotherapy patients: improvements in hemoglobin and quality of life are similar to threetimes-weekly dosing. J Clin Oncol, 19:2875-82.

Glaspy J, Bukowski R, Steinberg D, et al. 1997. Impact of therapy with epoetin alfa on clinical outcomes in patients with nonmyeloid malignancies during cancer chemotherapy in community oncology practice. Procrit Study Group. J Clin Oncol, 15:1218-34.

Groopman JE, Itri LM. 1999. Chemotherapy-induced anemia in adults: incidence and treatment. J Natl Cancer Inst, 91:1616-34.

Hagberg A, Barbany G, Landegren U, et al. 2003. Beta-globin mRNA increases rapidly during erythropoietin treatment. Scand J Clin Lab Invest, 63:239-45.

Hedenus M, Adriansson M, Miguel JS, et al. 2003. Efficacy and safety of darbepoetin alfa in anaemic patients with lymphoproliferative malignancies: a randomized, double-blind, placebo-controlled study. $\mathrm{Br} \mathrm{J}$ Haematol, 122:394-403.

Hedenus M, Birgegård G, Näsman P, et al. 2007. Addition of intravenous iron to epoetin beta increases hemoglobin response and decreases epoetin dose requirement in anemic patients with lymphoproliferative malignancies: a randomized multicenter study. Leukemia, 21:627-32.

Henke M, Laszig R, Rübe C, et al. 2003. Erythropoietin to treat head and neck cancer patients with anaemia undergoing radiotherapy: randomised, double-blind, placebo-controlled trial. Lancet, 362(9392):1255-60.

Henry D, Abels R, Larholt K. 1995. Prediction of response to recombinant human erythropoietin (r-HuEPO/epoetin-alpha) therapy in cancer patients. Blood, 85:1676-78.

Henry DH, Dahl NV, Auerbach M, et al. 2007. Intravenous ferric gluconate significantly improves response to epoetin alfa versus oral iron or no iron in anemic patients with cancer receiving chemotherapy. Oncologist, 12:231-42.

Hesketh PJ, Arena F, Patel D, et al. 2004. A randomized controlled trial of darbepoetin alfa administered as a fixed or weight-based dose using a front-loading schedule in patients with anemia who have non-myeloid malignancies. Cancer, 100:859-68.

Katodritou E, Speletas M, et al. 2004. Prediction of response of anemia to recombinant human erythropoietin in patients with multiple my eloma and lymphoma. Hematol J, 5(suppl 2):191.

Katodritou E, Terpos E, Zervas K, et al. 2007. Hypochromic erythrocytes (\%): a reliable marker for recognizing iron-restricted erythropoiesis and predicting response to erythropoietin in anemic patients with myeloma and lymphoma. Ann Hematol, 86:369-76.

Kyle RA. 1975. Multiple myeloma: review of 869 cases. Mayo Clin Proc, 50:29-40.

Leyland-Jones B, Semiglazov V, Pawlicki M, et al. 2005. Maintaining normal hemoglobin levels with epoetin alfa in mainly nonanemic patients with metastatic breast cancer receiving first-line chemotherapy: a survival study. J Clin Oncol, 23:5960-72.

Littlewood TJ, Bajetta E, Nortier JW, et al.; Epoetin Alfa Study Group. 2001. Effects of epoetin alfa on hematologic parameters and quality of life in cancer patients receiving nonplatinum chemotherapy: results of a randomized, double-blind, placebo-controlled trial. J Clin Oncol, 19:2865-74.

Ludwig H, Strasser K. 2001. Symptomatology of anemia. Semin Oncol, 28(2 Suppl 8):7-14.
Ludwig H, Van Belle S, Barrett-Lee P, et al. 2004. The European Cancer Anemia Survey (ECAS): a large, multinational, prospective survey defining the prevalence, incidence, and treatment of anemia in cancer patients. Eur J Cancer, 40:2293-306.

Lyman GH, Glaspy J. 2006. Are there clinical benefits with early erythropoietic intervention for chemotherapy-induced anemia? A systematic review. Cancer, 106:223-33.

Macdougall IC, Tucker B, Thompson J, et al. 1996. A randomized controlled study of iron supplementation in patients treated with erythropoietin. Kidney Int, 50:1694-99.

Macdougall I. 1998. Merits of percentage of hypochromic red cells as a marker of functional iron deficiency. Nephrol Dial Transplant, 13:847-49.

Mercadente S, Gebbia V, Marrazzo A, et al. 2000. Anemia in cancer: pathophysiology and treatment. Cancer Treat Rev, 25:303-11.

Miller CB, Jones RJ, Piantadosi S, et al. 1990. Decreased erythropoietin response in patients with the anemia of cancer. $N$ Engl $J$ Med, 322:1689-92.

Mittelman M, Zeidman A, Fradin Z, et al. 1997. Recombinant human erythropoietin in the treatment of multiple myeloma-associated anemia. Acta Haematol, 98:204-10.

Mittelman M, Neumann D, Peled A, et al. 2001. Erythropoietin induces tumor regression and antitumor immune responses in murine myeloma models. Proc Natl Acad Sci USA, 98:5181-6.

Mittelman M, Zeidman A, Kanter P, et al. 2004. Erythropoietin has an antimyeloma effect - a hypothesis based on a clinical observation supported by animal studies. Eur J Haematol, 72:155-65.

Moullet I, Salles G, Ketterer N, et al. 1998. Frequency and significance of anemia in non-Hodgkin's lymphoma patients. Ann Oncol, 9:1109-15

Nowrousian MR (Ed). 2002. Pathophysiology of cancer-related anemia. Recombinant human erythropoietin in clinical oncology. New York, Springer Medicine.

Nemeth E, Ganz T. 2006. Regulation of iron metabolism by hepcidin. Ann Rev Nutr, 26:323-42.

Osterborg A, Brandberg Y, Molostova V, et al. for the Epoetin Beta Hematology Study Group. 2002. Randomized, double-blind, placebocontrolled trial of recombinant human erythropoietin, epoetin beta, in hematologic malignancies. J Clin Oncol, 20(10):2486-94.

Osterborg A, Brandberg Y, Hedenus M. 2005. Impact of epoetin-beta on survival of patients with lymphoproliferative malignancies: long-term follow up of a large randomized study. Br J Haematol, 129:206-9.

Pangalis GA, Siakantaris M, Angelpolou MK, et al. 2002. Downstaging Rai stage III B-chronic lymphocytic leukemia patients with the administration of recombinant human erythropoietin. Hematologica, 87:500-6.

Punnonen K, Irjala K, Rajamaki A. 1997. Serum transferrin receptor and its ration to serum ferritin in the diagnosis of iron deficiency. Blood, 89:1052-57.

Quirt I, Robeson C, Lau CY, et al. 2001. Epoetin alfa therapy increases hemoglobin levels and improves quality of life in patients with cancerrelated anemia who are not receiving chemotherapy and patients with anemia who are receiving chemotherapy. J Clin Oncol, 19:4126-34.

Roy CN, Andrews NC. 2005. Anemia of inflammation: the hepcidin link. Curr Opin Haematol, 12:107-11.

Seidenfeld J, Piper M, Flamm C, et al. 2001. Epoetin treatment of anemia associated with cancer therapy: a systematic review and meta-analysis of controlled clinical trials. J Natl Cancer Inst, 93:1204-14.

Seidenfeld J, Piper M, Aronson N. 2002. Systematic review of controlled trials on erythropoietin to support evidence-based guidelines. Oncology (Williston Park), 16(9 Suppl 10):171-88.

Sepandj F, Jindal K, West M, et al. 1996. Economic appraisal of maintenance parenteral iron administration in treatment of anemia in chronic haemodialysis patients. Neprol Dial Transplant, 11:319-22.

Siakantaris MP, Angelopoulou MK, Vassilakopoulos TP, et al. 2000. Correction of disease related anemia of B-chronic lymphoproliferative disorders by recombinant human erythropoietin: maintenance is necessary to sustain response. Leuk Lymphoma, 40(1-2):141-7. 
Steensma DP, Molina R, Sloan JA, et al. 2006. Phase III study of two different dosing schedules of erythropoietin in anemic patients with cancer. $J$ Clin Oncol, 24:1079-89.

Straus DJ, Testa MA, Sarokhan BJ, et al. 2006. Quality-of-life and health benefits of early treatment of mild anemia: a randomized trial of epoetin alfa in patients receiving chemotherapy for hematologic malignancies. Cancer, 107:1909-17.

Thomas C, Kirschbaum A, Boehm D, et al. 2006. The diagnostic plot: a concept for identifying different states of iron deficiency and monitoring the response to epoetin therapy. Med Oncol, 23:23-36.

Tonelli M, Blake PG, Muirhead N, et al. 2001. Predictors of erythropoietin responsiveness in chronic hemodialysis patients. Asaio J, 47:82-5.
Van Belle S, Paridaens R, Evers G, et al. 2005. Comparison of proposed diagnostic criteria with FACT-F and VAS for cancer-related fatigue: proposal for use as a screening tool. Support Care Cancer, 13:246-54.

Vansteenkiste J. Wauters I. 2005. The use of darbepoetin alfa for the treatment of chemotherapy-induced anemia. Expert Opin Pharmacother, 6:429-40.

Witzig TE, Silberstein PT, Loprinzi CL, et al. 2005. Phase III, randomized, double-blind study of epoetin alfa compared with placebo in anemic patients receiving chemotherapy. J Clin Oncol, 23:2606-17.

Yellen SB, Cella DF, Webster K, et al. 1997. Measuring fatigue and other anemia-related symptoms with the Functional Assessment of Cancer Therapy (FACT) measurement system. J Pain Symptom Manage, 13:63-74. 
